\title{
A CROWD-STRUCTURE INTERACTION MODEL TO ANALYZE THE LATERAL LOCK-IN PHENOMENON ON FOOTBRIDGES
}

\author{
JAVIER FERNANDO JIMÉNEZ-ALONSO ${ }^{1}$, ANDRES SÁEZ $^{2}$, ELSA CAETANO $^{3}$ \& ALVARO CUNHA ${ }^{3}$ \\ ${ }^{1}$ Department of Building Structures, Universidad de Sevilla, Spain. \\ ${ }^{2}$ Department of Continuum Mechanics and Structural Analysis, Universidad de Sevilla, Spain. \\ ${ }^{3}$ Department of Civil Engineering, FEUP, Porto, Portugal.
}

\begin{abstract}
In this paper a simplified biomechanical crowd-structure interaction model is proposed and validated in order to analyse the lateral lock-in phenomenon on real footbridges. The proposed crowd-structure interaction model is organized in three levels: (i) pedestrian-structure interaction; (ii) interaction among pedestrians in the crowd; and (iii) interaction between the crowd and the structure. To this end, first, the human-structure interaction of each pedestrian is modelled via a simplified two degrees of freedom system. Second, the interaction among pedestrians inside the crowd is simulated using a multi-agent model. The considered model simulates the movement of each pedestrian from the dynamic equilibrium of the different social forces that act on him/her. Finally, the crowd-structure interaction is achieved modifying the behaviour of the pedestrians depending on the comfort level experienced. For this purpose, the recommendations established by the French standards have been considered. The integration of the three levels in an overall model is achieved by the implementation of a predictivecorrective method. The performance of the proposed model is validated correlating the numerical and experimental dynamic response of the Pedro e Inês footbridge during the development of a lateral lock-in pedestrian test. As the first lateral natural frequency of the footbridge is inside the range that characterizes the walking pedestrian step frequency in lateral direction, numerical and experimental studies were performed to analyse its behaviour under pedestrian action. The agreement between the numerical and experimental results is adequate. However, further studies are recommended in order to generalize the proposed approach and facilitate its use during the design project of future footbridges. Keywords: crowd dynamics, footbridge, human-structure interaction, lateral lock-in, simplified biomechanical model
\end{abstract}

\section{INTRODUCTION}

During the last 20 years numerous models have been proposed in order to analyse the lateral lock-in phenomenon on footbridges [1]. Although all these models have shed light in order to simulate numerically this phenomenon, they present the limitation of only taking into simplified account the modification of the modal parameters of the structure due to the pedestrian-structure interaction. The consideration of this fact may improve the numerical estimations of the dynamic response of these structures under pedestrian flows [2, 3].

In order to overcome this limitation, a crowd-structure interaction model is proposed and validated, in this study, to simulate more accurately the lateral lock-in phenomenon on footbridges. The proposed model allows estimating the change of the modal properties of footbridges under pedestrian flows. As consequence of this fact, the model allows a more accurate numerical analysis of the lateral lock-in phenomenon on real footbridges. The performance of the proposal is validated correlating the experimental and numerical lock-in phenomenon on a real footbridge, Pedro e Inês footbridge (Coimbra, Portugal) [4, 5].

The manuscript is organized as follows. The lateral crowd-structure interaction model is discussed in section 2; the Pedro e Inês footbridge is described in section 3; section 4 is dedicated to correlate the experimental and numerical results of a lateral lock-in pedestrian test 
performed on the footbridge; and finally, section 5 presents the main conclusions of this research.

\section{CROWD-STRUCTURE INTERACTION MODEL IN LATERAL DIRECTION}

The lateral crowd-structure interaction phenomenon is simulated using two individual sub-models: (i) a pedestrian-structure interaction sub-model and (ii) a crowd sub-model. In the first sub-model, each pedestrian is modelled as a TDOF (two degree of freedom) system and the dynamic behaviour of the structure is simulated via its modal parameters. In the second sub-model, the crowd is simulated using a behavioural model that provides a description of the individual pedestrian position, walking pedestrian velocity, step pedestrian frequency and phase between pedestrians. In order to consider the modification of the pedestrian behaviour associated with the vibration level, two additional conditions have been included in this sub-model: (i) a reduction factor, that affects the pedestrian velocity according to the comfort level experienced by each pedestrian; and (ii) a lock-in threshold, that modifies the step pedestrian frequency and the phase among pedestrians in order to simulate the crowd-structure synchronization [2]. In the following subsections, each sub-model and their interactions are described briefly. A more detailed presentation of the model may be found in Ref. [6, 7].

\subsection{Pedestrian-structure interaction in the lateral direction}

The proposed model follows from the application of dynamic equilibrium equations [8] to a TDOF-system (Fig. 1) with sprung $\left(m_{a, l a t}\right)$ and unsprung masses $\left(m_{s, l a t}\right)$.

The following coupled equation system may be obtained considering the dynamic balance of the proposed system, structure and pedestrian model.

$$
\begin{gathered}
M_{i} \ddot{y}_{i}+C_{i} \dot{y}_{i}+K_{i} y_{i}=\varphi_{\text {num }_{-} i}\left(v_{p x} \cdot t\right) \cdot F_{\mathrm{int}} \\
m_{a, \text { lat }} \ddot{y}_{a}+c_{p, l a t}\left(\dot{y}_{a}-\dot{y}_{s}\right)+k_{p, l a t}\left(y_{a}-y_{s}\right)=0 \\
m_{s, \text { lat }} \ddot{y}_{s}+c_{p, l a t}\left(\dot{y}_{s}-\dot{y}_{a}\right)+k_{p, l a t}\left(y_{s}-y_{a}\right)=F_{p, l a t}-F_{\mathrm{int}}
\end{gathered}
$$

where

$m_{a, l a t}, m_{s, l a t}$ and $m=m_{s, l a t}+m_{a, l a t}$ are the lateral pedestrian sprung mass, unsprung mass and the total pedestrian mass $[\mathrm{kg}]$, respectively. A medium pedestrian weight of $70 \mathrm{~kg}$, according to Synpex guidelines [9], was considered in this study.

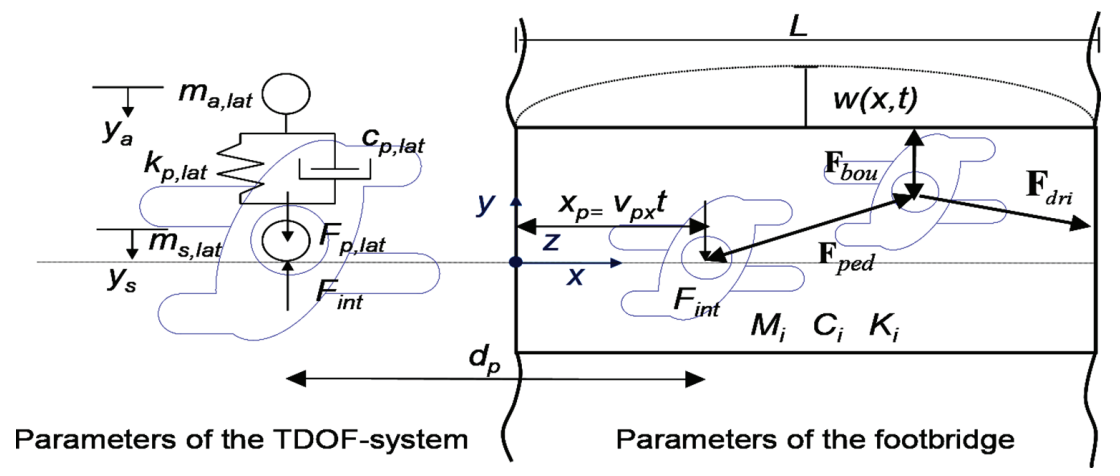

Figure 1: Biomechanical pedestrian-structure interaction model in lateral direction [6, 7]. 
$y_{i}, y_{a}$ and $y_{s}$ are the lateral displacement of the vibration mode $\mathrm{i}$ of the footbridge, the pedestrian sprung mass and the unsprung mass [m].

$k_{p, l a t}$ and $c_{p, l a t}$ are the lateral pedestrian stiffness $[\mathrm{N} / \mathrm{m}]$ and damping [sN/m].

$F_{p, l a t}$ and $F_{\text {int }}$ are the lateral pedestrian walking and pedestrian-structure interaction forces [N].

$M_{i}, C_{i}$ and $K_{i}$ are the modal mass $[\mathrm{kg}]$, modal damping [sN/m] and modal stiffness [N/m] of the vibration mode $\mathrm{i}[\mathrm{kg}]$.

$\varphi_{\text {num } i}$ is the lateral component of the numerical vibration mode $\mathrm{i}$.

$v_{p x}$ is the longitudinal component of the pedestrian velocity vector $[\mathrm{m} / \mathrm{s}]$.

$t$ is the time [sec.].

$d_{p}$ is the distance among pedestrians [m].

$w(x, t)$ is the lateral displacement of the footbridge at the position $x[\mathrm{~m}]$.

$L$ is the length of the footbridge [m].

The lateral pedestrian walking force can be expressed, according to the Synpex guidelines [9], in terms of Fourier series decomposition as:

$$
F_{p, l a t}=m \cdot g \cdot \sum_{i=1}^{3} \alpha_{i, l a t} \cdot \sin \left(\pi \cdot i \cdot f_{s} \cdot t-\phi_{i, l a t}-\varphi_{p}\right)
$$

where

$g$ is the acceleration of gravity.

$\alpha_{i, \text { lat }}$ is the Fourier coefficient of the $i t h$ harmonic for lateral forces or lateral dynamic load factor (LDLF).

$f_{s}$ is the pedestrian step frequency $[\mathrm{Hz}]$.

$\phi_{i, l a t}$ and $\varphi_{p}$ are the phase shift of the $i$ th harmonic of the lateral pedestrian force and among pedestrians respectively [rad].

Applying at the contact point the compatibility conditions of displacements, velocity and acceleration between the footbridge and the TDOF-system that models the pedestrian, the above relations, eqns (1-3), may be organized in a matrix form.

$$
\mathbf{M}(t) \cdot \ddot{\mathbf{y}}(t)+\mathbf{C}(t) \cdot \dot{\mathbf{y}}(t)+\mathbf{K}(t) \cdot \mathbf{y}(t)=\mathbf{F}(t)
$$

For a group of $k$ pedestrians (Fig. 1), we may further represent each one by the above TDOF-system. Thus, a system of $n+k$ differential equations will need to be solved (being $n$ the number of considered vibration modes). Finally, for the numerical evaluation of the resulting system, the $\beta$-Newmark integration family method is used, considering as parameters $\beta=1 / 4$ and $\gamma=1 / 2$, thus ensuring an unconditionally stable system.

\subsection{Parameters of the pedestrian-structure interaction model}

The parameters of the pedestrian-structure interaction model (pedestrian modal parameters and walking pedestrian lateral force) have been estimated experimentally via the solution of two inverse problems based on the results of several pedestrian tests performed on a real footbridge $[6,7]$. According to the results of this study, the following Gaussian distributions are established in Table 1 for the parameters that characterize the pedestrian-structure interaction model $(N(\mu, \sigma)$, being $\mu$ the mean value and $\sigma$ the standard deviation).

Once determined the pedestrian-structure interaction parameters, the definition of the proposed model is complete. 
Table 1: Parameters of the pedestrian-structure interaction model [6, 7].

Modal Parameters

\begin{tabular}{lll}
\hline Definition & Parameter & Value \\
\hline Pedestrian lateral sprung mass & $m_{a, \text { lat }}$ & $N(73.216,2.736) \%$ \\
Pedestrian lateral damping ratio & $\zeta_{p, \text { lat }}$ & $N(49.116,5.405) \%$ \\
Pedestrian lateral natural frequency & $f_{p, \text { lat }}$ & $N(1.201,0.178) \mathrm{Hz}$ \\
\hline Walking Pedestrian Lateral force & & \\
\hline Definition & Parameter & Value \\
\hline First LDLF & $\alpha_{1, \text { lat }}$ & $N(0.086,0.017)$ \\
Second LDLF & $\alpha_{2, \text { lat }}$ & $N(0.094,0.009)$ \\
Third LDLF & $\alpha_{3, \text { lat }}$ & $N(0.040,0.019)$ \\
First lateral phase shift & $\phi_{1, \text { lat }}$ & $0^{\circ}$ \\
Second lateral phase shift & $\phi_{2 \text { lat }}$ & $0^{\circ}$ \\
Third lateral phase shift & $\phi_{3, \text { lat }}$ & $0^{\circ}$ \\
\hline
\end{tabular}

\subsection{Modelling the crowd behaviour}

The proposed multi-agent model that simulates the behaviour of the crowd consists in the sum of three partial forces that represent the different influences that the pedestrians undergo when interacting in a crowd [10]. Thus, the resultant social force, $\mathbf{F}_{p c i}$, is defined as (Fig. 1):

$$
\mathbf{F}_{p c i}=\mathbf{F}_{d r i}+\mathbf{F}_{p e d}+\mathbf{F}_{b o u}
$$

where

$\mathbf{F}_{d r i}$ is the driving force that reflects the motivation of each pedestrian to reach his desired destination $[\mathrm{N}]$.

$\mathbf{F}_{\text {ped }}$ is the repulsive force, which simulates the interaction among pedestrians [N].

$\mathbf{F}_{\text {bou }}$ is the repulsive force, which simulates the interaction among the pedestrians and the boundaries [N].

In order to simulate the pedestrians flow, three parameters must be defined: (i) the pedestrian density $\left[\mathrm{P}=\right.$ Person $\left./ \mathrm{m}^{2}\right]$, (ii) the value of the desired pedestrian velocity, and (iii) the distance between pedestrians. In the present study the pedestrian density is established depending on the expected pedestrian traffic according to the Synpex guidelines [9]. The values of the desired velocity of each pedestrian are obtained from the pedestrian step frequencies, defined according to the Gaussian distributions proposed by Zivanovic et al. [11], $N(1.87,0.186) \mathrm{Hz}$. Subsequently each assigned step frequency is converted into the desired velocity using the empirical relation proposed by Bertram and Ruina [12]. Additionally, the original distance among pedestrians is calculated assuming a rectangular-shaped mesh of pedestrians and the considered pedestrian density. Finally, the phase shift among pedestrians, $\varphi_{p}$, is determined initially adopting a Poisson distribution [8]. Subsequently, this parameter is modified via the crowd-structure interaction. 


\subsection{Crowd-structure interaction}

The maximum lateral acceleration experienced by each pedestrian may be compared against the acceleration threshold values established by the Synpex guidelines [9] in order to modify the individual pedestrian behaviour inside the crowd. Two types of thresholds have been considered in the model for this purpose: (i) comfort thresholds and (ii) a lateral lock-in threshold. First, in order to reflect in the model the change of the behaviour of each pedestrian due to the modification of his comfort level, a behavioural factor has been applied to the pedestrian velocity. According to the results provided by previous studies [3], minimum and maximum comfort thresholds of $0.20 \mathrm{~m} / \mathrm{s}^{2}$ and $2.10 \mathrm{~m} / \mathrm{s}^{2}$, respectively, have been selected in the model. In this manner, if the lateral acceleration of each pedestrian exceeds the minimum value, the pedestrian reduces its velocity, stopping in case the maximum value is overcome. Second, in order to take into account in the model the lateral lock-in phenomenon, the criterion provided by the French standard [13] is considered. If the lateral acceleration experienced by each pedestrian is above $0.15 \mathrm{~m} / \mathrm{s}^{2}$ and his step frequency is within $\pm 10 \%$ of the lateral natural frequency of the structure, both his step frequency and phase shift are modified to match the natural frequency of the footbridge, synchronizing the movements of the pedestrian and the structure.

\section{DESCRIPTION OF PEDRO E INÊS FOOTBRIDGE}

The Pedro e Inês footbridge is located at Coimbra (Portugal). The total length of the structure is $274.5 \mathrm{~m}$, configured by one central arch of $110 \mathrm{~m}$, two lateral semi-arches of $64 \mathrm{~m}$ and two transition spans of 30.5 and $6 \mathrm{~m}$ (Fig. 2a). The main feature of the footbridge is the antisymmetrical configuration of the deck and the arches with respect to the longitudinal axis of the structure. The deck is a concrete-steel composite box-girder with a variable width between 4 and $8 \mathrm{~m}$, which generates a panoramic square at mid-span of the footbridge (Fig. 2b). Even
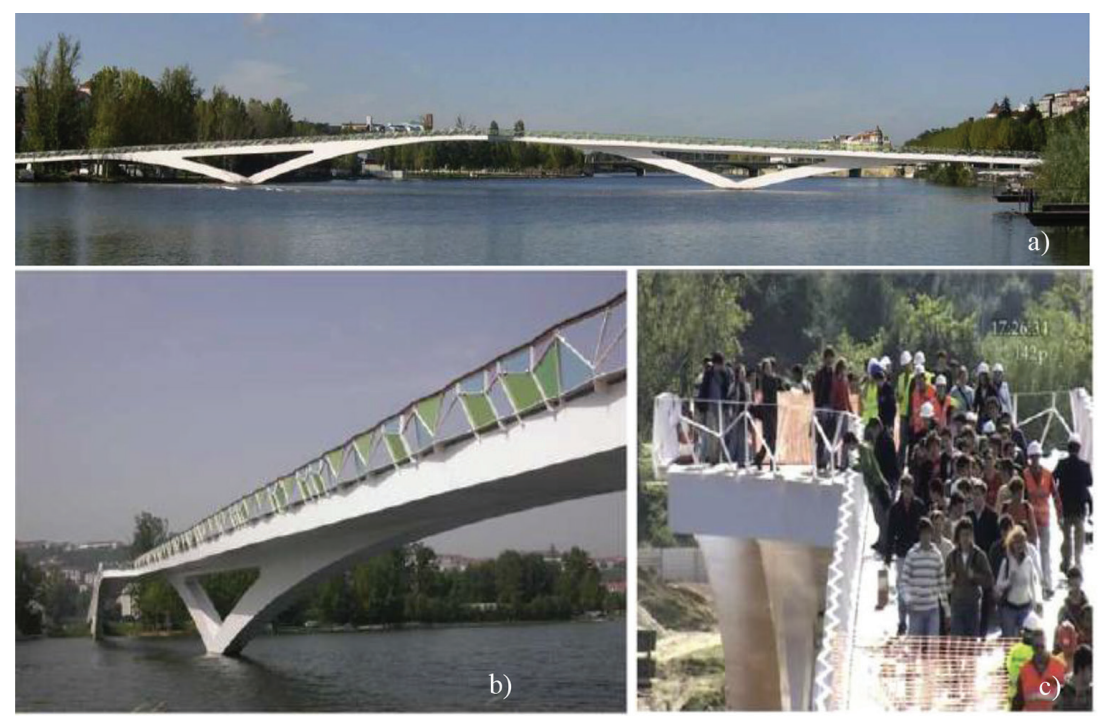

Figure 2: (a) General view of Pedro e Inês footbridge; (b) Perspective of the footbridge and (c) experimental lateral lock-in pedestrian test on this footbridge [4, 5]. 
during the design phase, numerical studies indicated that the structure was prone to vibrations induced by pedestrians in the lateral direction. This fact motivated the development of a precise and detailed work for the experimental assessment of the dynamic response and the implementation of a control system in order to guarantee an adequate comfort level of the footbridge. This work was performed and reported by Caetano et al. [4, 5] and its results have been used in this paper in order to validate the proposed lateral crowd-structure interaction model.

The footbridge presented a first lateral vibration mode with an experimental natural frequency of $0.91 \mathrm{~Hz}$, with an associated damping ratio of $0.55 \%$, which was easily excited by the pedestrian flows. In order to determine experimentally the number of pedestrians that originates the lateral lock-in phenomenon, an experimental test was performed. The result of this experimental test, reported in the literature $[4,5]$, has been correlated with the numerical simulation based on the implementation of the proposed lateral crowd-structure interaction model on an updated finite element model [14] of the Pedro e Inês footbridge.

\section{LATERAL LOCK-IN PEDESTRIAN TEST ON THE PEDRO E INÊS FOOTBRIDGE}

In the experimental lateral lock-in pedestrian test, the lateral acceleration, $a_{\text {lat }}$, at mid-span of the footbridge under the passage of different group of pedestrians was recorded (Fig. 2c). A graphical representation of the maximum lateral acceleration at this position versus the number of pedestrians on the footbridge (Fig. 3) allows for identifying the instability situation associated with the lateral lock-in phenomenon. As it is illustrated in Fig. 3, the number of pedestrians that originates the beginning of the lateral lock-in phenomenon is around 75 .

Subsequently, a numerical lateral lock-in pedestrian test based on the proposed lateral crowd-structure interaction model was performed. Each considered group of pedestrians were simulated considering as initial spatial distribution a rectangular-shaped grid with an initial distance among pedestrians of $0.50 \mathrm{~m}$ in the longitudinal direction and an equidistant distribution in the lateral direction. During the numerical simulation, each considered group of pedestrians walks freely along the footbridge. The number of pedestrians in each group increases gradually between 15 and 85 in increments of 5 . The maximum numerical lateral acceleration at mid-span versus the number of pedestrians on the footbridge is shown in Fig. 3.

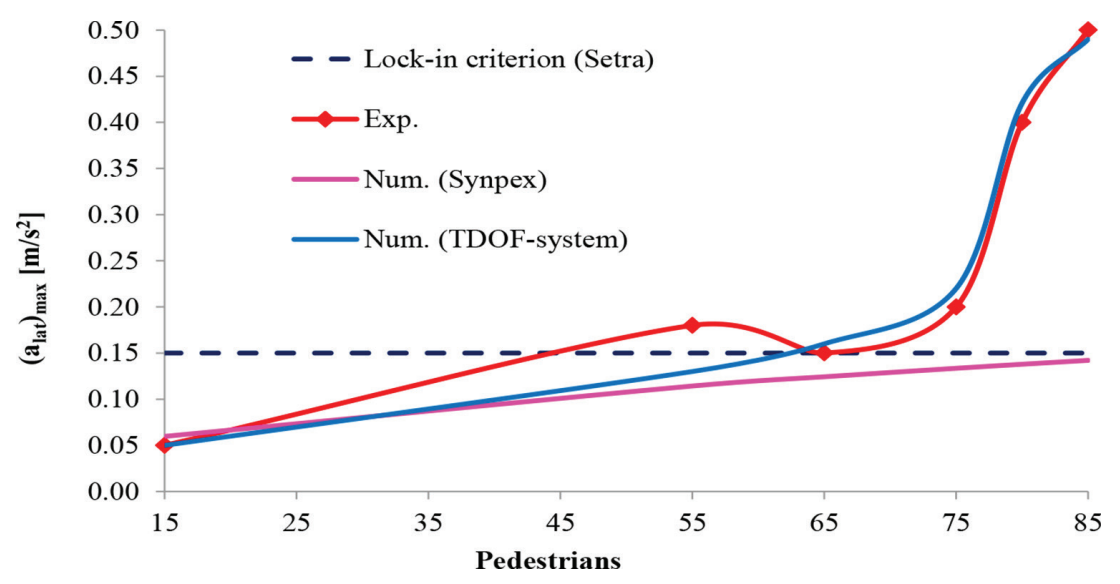

Figure 3: Experimental $[4,5]$ and numerical variation of the maximum lateral acceleration, $\left(a_{\text {lat }}\right)_{\max }$. 
As Fig. 3 shows, the correlation between the experimental lateral maximum accelerations and the numerically estimated maximum values is adequate. Additionally, the estimation of the numerical maximum acceleration obtained, applying the methodology proposed by the Synpex guidelines [9], is also shown in Fig. 3. It is clear from Fig. 3 that the proposed model allows obtaining a more accurate numerical analysis of the lateral lock-in phenomenon than these standards. The lateral lock-in criterion established by French standards [13] is also illustrated for reference in Figure 3.

\section{CONCLUSIONS}

In this paper, a crowd-structure interaction model is proposed and validated to analyse the lateral lock-in phenomenon on footbridges under pedestrian flows. The proposed model is organized in two sub-models: (i) a pedestrian-structure interaction and (ii) a crowd submodel. The pedestrian-structure interaction sub-model is defined in terms of a TDOF-system. The crowd sub-model is defined in terms of a multi-agent model based on the social force model. The interaction between the two sub-models is achieved by imposing two behavioural conditions: a comfort and lateral lock-in thresholds. According to these requirements if certain acceleration limits are exceeded the affected pedestrians modify their behaviour. The proposed model is formulated under the following hypotheses: (i) constant parameters of the pedestrian-structure interaction model; (ii) dynamic effects associated to the change of the pedestrian velocity neglected, and (iii) the interaction problem only considers the walking action. The proposed model has been implemented in order to analyse the correlation between the experimental and numerical lateral lock-in phenomenon on a real footbridge, the Pedro e Inês footbridge (Coimbra, Portugal). The results obtained from the proposed model are adequate, allowing the accurate prediction of the lateral lock-in phenomenon on this footbridge and consequently the validation of the proposal. The proposed model may be an useful tool to analyse the lateral lock-in phenomenon on footbridges during the design phase. Nevertheless, further studies, additional pedestrian and crowd tests on different footbridges, are recommended in order to better characterize the lateral lock-in phenomenon on real footbridges.

\section{ACKNOWLEDGEMENTS}

This work was partially funded by the Spanish Ministry for Science under research project DPI2014-53947-R.

\section{REFERENCES}

[1] Ingólfsson, E.T., Georgakis, C.T., Ricciardelli, F. \& Jönsson, J., Experimental identification of pedestrian-induced lateral forces on footbridges. Journal of Sound and Vibration, 330, pp. 1265-1284, 2011. https://doi.org/10.1016/j.jsv.2010.09.034

[2] Carrol, S.P., Owen, J.S. \& Hussein, M.F.M., Modelling crowd-bridge dynamic interaction with a discretely defined crowd. Journal of Sound and Vibration, 331, pp. 2685-2709, 2012. https://doi.org/10.1016/j.jsv.2012.01.025

[3] Bocian, M., Macdonald, J.H.G. \& Burn J.F., Biomechanically inspired modelling of pedestrian-induced forces on laterally oscillating structures. Journal of Sound and Vibration Sound, 331, pp. 3914-3929, 2012. https://doi.org/10.1016/j.jsv.2012.03.023 
[4] Caetano, E., Cunha, A., Magalhães, F. \& Moutinho, C., Studies for controlling humaninduced vibration of the Pedro e Inês footbridge, Portugal. Part 1: Assessment of dynamic behaviour. Engineering Structures, 32, pp. 1069-1081, 2010.

https://doi.org/10.1016/j.engstruct.2009.12.034

[5] Caetano, E., Cunha, A., Moutinho, C. \& Magalhães, F., Studies for controlling humaninduced vibration of the Pedro e Inês footbridge, Portugal. Part 2: Implementation of tuned mass dampers. Engineering Structures, 32, pp. 1082-1091, 2010.

https://doi.org/10.1016/j.engstruct.2009.12.033

[6] Jiménez-Alonso, J.F., Proposal and calibration of a biodynamic model of human-structure interaction by the resolution of the inverse dynamic problem: application to pedestrian bridges, PhD Thesis, University of Seville, 2015.

[7] Jiménez-Alonso, J.F., Sáez, A., Caetano, E. \& Cunha, A., Lateral crowd-structure interaction model to analyse the change of the modal properties of footbridges. Submitted for publication.

[8] Jiménez-Alonso, J.F., Sáez, A., Caetano, E. \& Magalhães, F., Vertical crowd-structure interaction model to analyse the change of the modal properties of a footbridge. Journal of Bridge Engineering ASCE, 21(8), pp. C4015004/1-19, 2016.

https://doi.org/10.1061/(asce)be.1943-5592.0000828

[9] Butz, C.H., Heinemeyer, C.H., Goldack, A., Keil, A., Lukic, M., Caetano, E. \& Cunha, A., Advanced load models for Synchronous Pedestrian Excitation and Optimised Design Guidelines for Steel Footbridges (SYNPEX). RFCS-Research Project RFS-CR-03019. 2007.

[10] Helbing, D. \& Molnár, P., Social force model for pedestrian dynamics. Physical Review, 51(5), pp. 4282-4286, 1995.

https://doi.org/10.1103/physreve.51.4282

[11] Zivanovic, S., Pavic, A. \& Ingolfsson, E., Modelling spatially unrestricted pedestrian traffic on footbridges. Journal of Structural Engineering, 136(10), pp. 1296-1308, 2010.

https://doi.org/10.1061/(asce)st.1943-541x.0000226

[12] Bertram, J.E.A. \& Ruina, A., Multiple walking speed-frequency relations are predicted by constrained optimization. Journal of Theoretical Biology, 209(4), pp. 445-453, 2001.

https://doi.org/10.1006/jtbi.2001.2279

[13] SETRA/AFGC. Guide méthodologique passerelles piétonnes (Technical Guide Footbridges: Assessment of vibration behaviour of footbridge under pedestrian loading). SETRA, 2006.

[14] Mottershead J.E., Link M. \& Friswell M.I., The sensitivity method in finite element model updating: a tutorial. Mechanical System and Signal Processing, 25, pp. 22752296, 2011.

https://doi.org/10.1016/j.ymssp.2010.10.012 